\title{
In silico analysis of signal peptides for secretory production of a- amylase in Bacillus subtilis
}

\author{
Marzieh Asadia, Mortaza Taheri-Anganeha, Zeinab Jamalib, Seyyed Hossein Khatamic, Cambyz \\ Irajie $^{\mathrm{a}}$, Amir Savardashtaki ${ }^{\mathrm{a}, \mathrm{d}^{*}}$, Younes Ghasemi ${ }^{\mathrm{a}, \mathrm{d}, \mathrm{e}}$ \\ ${ }^{a}$ Department of Medical Biotechnology, School of Advanced Medical Sciences and Technologies, Shiraz University of Medical Sciences, Shiraz, Iran \\ ${ }^{b}$ Cardiovascular Research Center, Shiraz University of Medical Sciences, Shiraz, Iran \\ 'Department of Biochemistry, School of Medicine, Shiraz. University of Medical Sciences, Shiraz, Iran \\ ¿Pharmaceutical Sciences Research Center, Shiraz. University of Medical Sciences, Shiraz, Iran \\ ${ }^{e}$ Department of Pharmaceutical Biotechnology, School of Pharmacy, Shiraz. University of Medical Sciences, Shiraz, Iran
}

Received 31st May 2019 / Accepted 24th July 2019

\begin{abstract}
Amylases are important commercial enzymes and have a broad application in industrial processes and medicine. Gram-positive bacteria such as Bacillus subtilis are possible host organisms for $\alpha$ amylases secretory production. Secretion of $\alpha$-amylases to the culture medium versus intracellular production has several advantages such as prevention of inclusion bodies accumulation, higher product stability and solubility. Signal peptides are considered as one of the most essential elements for successful secretory synthesis of the recombinant proteins. Therefore, by the selection of an efficient signal peptide, secretion of the recombinant protein can be enhanced. The goal of this investigation was the in silico evaluation of several peptides to find the most suitable leader peptides for secretory production of $\alpha$ amylase in B. subtilis. In present work, 30 signal peptides were selected, and numerous online servers such as SignalP, ProtParam, SOLpro, PRED-TAT and ProtComp was used for investigation of suitable signal peptides. According to in silico predictions all other signal peptides connected to $\alpha$-amylase were stable and soluble except PPBD_BACSU. PPBD_BACSU because of having D-score below cut-off could not be recognized as a suitable signal peptide for $\alpha$-amylase. Computational analysis identified QOX2_BACSU may direct protein into transmembrane location and was ignored. All 28 remained were predicted as secretory signal peptides which can excrete protein out of the bacteria. The signal peptides recommended by the present study are valuable for rational designing of secretory soluble $\alpha$-amylase. Although, such information can be useful for future experimental production of these mentioned secretory proteins.
\end{abstract}

Keywords: $\alpha$-Amylase, in silico, recombinant protein, Bacillus subtilis, signal peptide

\section{INTRODUCTION}

Recombinant proteins, including commercial recombinant enzymes and biopharmaceutical proteins, have a multibillion-dollar sale per year (Freudl, 2018). $\alpha$-Amylases are important enzymes in market that comprise $30 \%$ of the world's enzymes market (Mobini-Dehkordi and Javan, 2012). In medicine, these enzymes are used to treat pancreatic insufficiency. Patients with cystic fibrosis can also be treated with enzyme- replacement therapies such as $\alpha$-amylase to prevent malnutrition because in cystic fibrosis disease the secretion of $\alpha$-amylase is obstructed (Li and Somerset, 2014, Azzopardi et al., 2016). These enzymes have a broad application in industrial processes, like the textile industry, paper, food, detergent and pharmaceutical field (Nascimento, 2013). The utilization of these biocatalysts in food industries improves the quality of

\footnotetext{
*Author for correspondence: Amir Savardashtaki, Department of Medical Biotechnology, School of Advanced Medical Sciences and Technologies, Shiraz University of Medical Sciences, Shiraz, IR Iran. Email dashtaki63@gmail.com
} 
products while reducing the use of different chemical materials, thereby helping the consumers' health (Mehta and Satyanarayana, 2016). Additionally, for reduction of processing time, cost-effectiveness, low energy consumption, nontoxicity and economic efficiency, the application of $\alpha$-amylases in various industries is increasing (Singh et al., 2016).

$\alpha$-Amylases grouped into the GH-13 family of glycosyl hydrolases are extracellular enzymes which randomly break the $\alpha$ (1-4) connection between two contiguous glucose units in the linear amylose chain and finally produce glucose, maltose, and maltotriose units. $\alpha$-Amylases can be synthesized by various living sources, including microorganisms, plants, and animals. However, bacterial and fungal enzymes have more commercial and industrial application due to their high efficiency and resistance to $\mathrm{pH}$ and temperature (Nascimento, 2013). However, it seems that bacterial sources are a better choice due to their stability at higher temperatures (Kindle, 1983). Among bacillus species, Bacillus licheniformis is known to be a good producer of thermostable $\alpha$-amylases (Schallmey et al., 2004).

Many different expression systems exist for the recombinant production of $\alpha$-amylase protein including different pro- and eukaryotic expression systems. Even though, for using as expression hosts, bacteria are more interesting (Freudl, 2018).

Escherichia coli (E. coli) is one of the most broadly applied hosts for the recombinant proteins synthesis but it cannot excrete proteins out of the cell. So, overexpression of proteins in it usually results in inclusion body formation or protein aggregation in the cytoplasm, which requires sophisticated refolding mechanisms to achieve biologically active proteins and consequently result in decreasing of active proteins yields (Cui et al., 2017, Wang et al., 2016). Therefore, there is some benefits for recombinant proteins secretion into extracellular medium, such as prevention of inclusion bodies accumulation, reduction or even alleviation of toxic effect that applied by target proteins, correct construction of disulfide bonds that are necessary for the final conformations, and simplification of product recovery (Freudl, 2018, Anné et al., 2016).

Gram-positive bacteria are considered as potential hosts for recombinant protein secretion. This type of bacteria contains only one lipid bilayer with a broad peptidoglycan cell wall and export target protein directly into the culture medium that is an ideal condition for correct folding of recombinant proteins (Green and Mecsas, 2016).

Bacillus subtilis is a gram-positive, safe bacterial strain that has a generally recognized as safe (GRAS) condition with an innate capability to excrete proteins into the medium (Zobel et al., 2015). The most significant normal secretion pathways in $B$. subtilis are twin-arginine translocation (Tat) pathway, Sec pathway and ATP-binding cassette $(\mathrm{ABC})$ pathway (Tjalsma et al., 2004, Anné et al., 2016). Signal peptides mark the proteins to follow the appropriate secretion pathway (Owji et al., 2018). Three separate domains of $\mathrm{H}, \mathrm{N}$, and $\mathrm{C}$ are observed in signal peptides. The $\mathrm{C}$ domain containing the cleavage site for the signal peptidase while the $h$ and $n$ regions are necessary for efficient protein transferring (Freudl, 2018, Tjalsma et al., 2004).

Since the secretion of a given recombinant protein can be enhanced by the selection of an efficient signal peptide, acquiring the most appropriate one for a given target protein is a critical step of an efficient secretory production procedure (Guan et al., 2016).

Despite different studies and deep considerations, there is no assurance in selecting a suitable signal sequence and confirm efficient secretion of the recombinant protein experimentally, and it needs a trial-and-error approach. One alternative strategy is employing the bioinformatics tools to perform In silico studies (Taheri-Anganeh et al., 2019). For example, numerous online servers such as SignalP, ProtParam, SOLpro, PRED-TAT and ProtComp are used for prediction of the best signal peptides (Mousavi et al., 2017, Negahdaripour et al., 2017). Thus, in our work, an in-silico approach is chosen to analyze several signal peptides for acquiring the most suitable candidates for secretory synthesis of $\alpha$-amylase in B. subtilis.

\section{MATERIALS AND METHODS}

Dataset collection. In the first step of the research, the primary structure of Bacillus licheniformis $\alpha$-amylase was retrieved from the 
UniProt server at http://www.uniprot.org/. In the second step, the amino acid sequences of the prokaryotic signal peptides were gained from the Signal Peptide Website at http://www.signal peptide.de/and validated by UniProt.

The prediction of cleavage site location of the signal peptides. There are several online servers for signal peptides prediction and determination of their cleavage site location. SignalP server at http://www.cbs.dtu.dk/services/SignalP/, was used to evaluate the presence of signal peptides and their cleavage sites locations by combining several artificial neural networks (Petersen et al., 2011).

\section{Signal peptides physicochemical aspects} prediction. ProtParam, as a part of ExPASy, is online at https://web.expasy.org/protparam/, was used for the computation of various physicochemical properties of the signal peptides, such as molecular weight (MW), isoelectric point $(\mathrm{pI})$, aliphatic index, instability index, positively charge residues and grand average of hydrophobicity (GRAVY) (Gasteiger et al., 2005).

Protein solubility prediction. SOLpro server at http://scratch.proteomics.ics.uci.edu/, was employed for solubility prediction. Using this server, the solubility of proteins can be analyzed, based on their amino acid sequences. SOLpro employs two-stage SVM structure based on primary sequences of proteins (Magnan et al., 2009).

\section{Evaluation of signal peptides secretion} pathway and sub-cellular localization. In silico prediction of secretion pathways of signal peptide connected fusions was implemented by "PREDTAT" online server (http://www.compgen.org/ tools/PRED-TAT). This server defines Sec or Tat pathway for secretion with a score in the 0-1 range. "ProtCompB" online server (http://www .softberry.com) was employed to evaluate subcellular location of fusion proteins (Bagos et al., 2010, Mohammadi et al., 2018).

\section{RESULTS AND DISCUSSION}

Retrieval of signal peptides and sequence definition. According to the Signal Peptide Website, 30 signal peptides including the natural Bacillus licheniformis $\alpha$-amylase and 29 other prokaryotic signal peptides were selected. 21 signal peptides were from gram-positive Bacilli, and nine signal peptides were selected from E. coli, which are listed in Table 1.

The prediction of cleavage site location. The presence of signal peptides and their cleavage site locations prediction are shown in Table 2. Dscore $>0.57$ for a sequence means that it is determined as a signal peptide. Based on these results, D-score of the PPBD_BACSU was under the cut-off (0.57) and was neglected for next steps of study.

Prediction of physicochemical aspects of signal peptides. Physicochemical properties of the signal peptides are shown in Table 3. All signal peptides were in the range of 19 and 41 amino acids in length. All the selected signal peptides had net positive charges of 1 to 6 based on ProtParam results. The highest GRAVY values belonged to QOX2_BACSU, CWBA_BACSU, and OMPC_ECOLI and GUB_BACAM respectively (2.242, 1.596, 1.552, and 1.508). The results showed that QOX2_BACSU, OMPC_ECOLI, CWBA_BACSU, and MPF_ECOLI, respectively $(198.46,171.90,160$, and 150.91) had the highest aliphatic index. Instability index represents that how much a signal peptide alone and fused to $\alpha$ amylase protein can be stable. Based on our results, $\alpha$-amylase fused with all signal peptides were predicted to be stable.

In silico evaluation of protein solubility. The solubility of the $\alpha$-amylase in fusion with different signal peptides is shown in Table 3 . Based on the results, $\alpha$-amylase in the fused form with all signal peptides was soluble. 
Table 1. Qualitative analyzes of protein spots detected of untreated, 5-Aza and TSA-treated HL-60.

\begin{tabular}{|c|c|c|c|c|c|}
\hline & $\begin{array}{l}\text { Uniprot } \\
\text { Accession } \\
\text { No. }\end{array}$ & $\begin{array}{l}\text { Signal } \\
\text { Peptide }\end{array}$ & Protein Name & Source & Amino Acid Sequence \\
\hline 1 & P06278 & $\begin{array}{c}\text { AMY_BAC } \\
\text { LI }\end{array}$ & Alpha amylase & $\begin{array}{c}\text { Bacillus } \\
\text { licheniformis }\end{array}$ & $\begin{array}{l}\text { MKQQKRLYARLLTLLFALIF } \\
\text { LLPHSAAAA }\end{array}$ \\
\hline 2 & P00808 & $\begin{array}{l}\text { BLAC_BA } \\
\text { CLI }\end{array}$ & Beta-lactamase & $\begin{array}{l}\text { Bacillus } \\
\text { licheniformis }\end{array}$ & $\begin{array}{l}\text { MKLWFSTLKLKKAAAVLLF } \\
\text { SCVALAG }\end{array}$ \\
\hline 3 & P16397 & $\begin{array}{c}\text { SUBF_BAC } \\
\text { SU }\end{array}$ & $\begin{array}{l}\text { Bacillopeptidase } \\
\text { F }\end{array}$ & Bacillus subtilis & $\begin{array}{l}\text { MRKKTKNRLISSVLSTVVISS } \\
\text { LLFPGAAGA }\end{array}$ \\
\hline 4 & Q02113 & $\begin{array}{c}\text { CWBA_BA } \\
\text { CSU }\end{array}$ & $\begin{array}{l}\text { Amidase } \\
\text { enhancer }\end{array}$ & Bacillus subtilis & $\begin{array}{l}\text { MKSCKQLIVCSLAAILLLIPS } \\
\text { VSFA }\end{array}$ \\
\hline 5 & P42111 & $\underset{\text { CSU }}{\text { YXAL_BA }}$ & $\begin{array}{l}\text { Uncharacterized } \\
\text { protein yxaL }\end{array}$ & Bacillus subtilis & $\begin{array}{l}\overline{\text { MVKSFRMKALIAGAAVAAA }} \\
\text { VSAGAVSDVPAAKVLQPTA } \\
\underline{\text { AYA }}\end{array}$ \\
\hline 6 & O31737 & $\begin{array}{l}\text { YLQB_BA } \\
\text { CSU }\end{array}$ & $\begin{array}{l}\text { Uncharacterized } \\
\text { protein ylqB }\end{array}$ & Bacillus subtilis & $\begin{array}{l}\text { MKKIGLLFMLCLAALFTIGF } \\
\text { PAQQADA }\end{array}$ \\
\hline 7 & P00691 & $\underset{\text { SU }}{\text { AMYBAC }}$ & Alpha-amylase & Bacillus subtilis & $\begin{array}{l}\text { MFAKRFKTSLLPLFAGFLLL } \\
\text { FHLVLAG }\end{array}$ \\
\hline 8 & P39116 & $\underset{U}{\text { PEL_BACS }}$ & Pectate lyase & Bacillus subtilis & $\begin{array}{l}\text { MKKVMLATALFLGLTPAGA } \\
\text { NA }\end{array}$ \\
\hline 9 & O07532 & $\begin{array}{l}\text { LYTF_BA } \\
\text { CSU }\end{array}$ & $\begin{array}{l}\text { Endopeptidase } \\
\text { lytF }\end{array}$ & Bacillus subtilis & $\begin{array}{l}\text { MKKKLAAGLTASAIVGTTLV } \\
\text { VTPAEA }\end{array}$ \\
\hline 10 & P42251 & $\begin{array}{l}\text { PPBD_BA } \\
\text { CSU }\end{array}$ & $\begin{array}{c}\text { Alkaline } \\
\text { phosphatase D }\end{array}$ & Bacillus subtilis & $\begin{array}{l}\text { MAYDSRFDEWVQKLKEESF } \\
\text { QNNTFDRRKFIQGAGKIAG } \\
\text { LSLGLTIAQSVGAFEVNA }\end{array}$ \\
\hline 11 & P34957 & $\begin{array}{c}\text { QOX2_BA } \\
\text { CSU }\end{array}$ & $\begin{array}{l}\text { Quinol oxidase } \\
\text { subunit } 2\end{array}$ & Bacillus subtilis & $\begin{array}{l}\text { MVIFLFRALKPLLVLALLTV } \\
\text { VFVLGG }\end{array}$ \\
\hline 12 & P04957 & $\underset{\text { SU }}{\text { GUB_BAC }}$ & Beta-glucanase & Bacillus subtilis & $\begin{array}{l}\text { MPYLKRVLLLLVTGLFMSLF } \\
\text { AVTATASA }\end{array}$ \\
\hline 13 & P00648 & $\begin{array}{l}\text { RNBR_BA } \\
\text { CAM }\end{array}$ & Ribonuclease & $\begin{array}{c}\text { Bacillus } \\
\text { amyloliquefaciens }\end{array}$ & $\begin{array}{l}\text { MMKMEGIALKKRLSWISVC } \\
\text { LLVLVSAAGMLFSTA }\end{array}$ \\
\hline 14 & P21130 & $\begin{array}{l}\text { SACB_BA } \\
\text { CAM }\end{array}$ & Levansucrase & $\begin{array}{l}\text { Bacillus } \\
\text { amyloliquefaciens }\end{array}$ & $\begin{array}{l}\text { MNIKKIVKQATVLTFTTALL } \\
\text { AGGATQAFA }\end{array}$ \\
\hline 15 & P00692 & $\begin{array}{c}\text { AMY_BAC } \\
\text { AM }\end{array}$ & Alpha-amylase & $\begin{array}{c}\text { Bacillus } \\
\text { amyloliquefaciens }\end{array}$ & $\begin{array}{l}\text { MIQKRKRTVSFRLVLMCTLL } \\
\text { FVSLPITKTSA }\end{array}$ \\
\hline 16 & P07980 & $\begin{array}{c}\text { GUB_BAC } \\
\text { AM }\end{array}$ & Beta-glucanase & $\begin{array}{l}\text { Bacillus } \\
\text { amyloliquefaciens }\end{array}$ & $\begin{array}{l}\text { MKRVLLILVTGLFMSLCGITS } \\
\text { SVSA }\end{array}$ \\
\hline 17 & Q05523 & $\underset{\text { CST }}{\text { DACA_BA }}$ & $\begin{array}{c}\text { D-alanyl-D- } \\
\text { alanine } \\
\text { carboxypeptidase } \\
\text { dacA }\end{array}$ & $\begin{array}{l}\text { Bacillus } \\
\text { stearothermophilus }\end{array}$ & $\begin{array}{l}\text { MRRRKQNWLFWLLSICLCL } \\
\text { TFGPFQQTVKA }\end{array}$ \\
\hline 18 & P31797 & $\begin{array}{c}\text { CDGT_BA } \\
\text { CST }\end{array}$ & $\begin{array}{c}\text { Cyclomaltodextri } \\
\mathrm{n} \\
\text { glucanotransfera } \\
\text { se }\end{array}$ & $\begin{array}{l}\text { Bacillus } \\
\text { stearothermophilus }\end{array}$ & $\begin{array}{l}\text { MRRWLSLVLSMSFVFSAIFIV } \\
\text { SDTQKVTVEA }\end{array}$ \\
\hline 19 & P06874 & $\begin{array}{c}\text { THER_BA } \\
\text { CST }\end{array}$ & Thermolysin & $\begin{array}{l}\text { Bacillus } \\
\text { stearothermophilus }\end{array}$ & $\begin{array}{l}\text { MNKRAMLGAIGLAFGLLAA } \\
\text { PIGASA }\end{array}$ \\
\hline 20 & P40943 & $\begin{array}{c}\text { XYN1_BA } \\
\text { CST }\end{array}$ & $\begin{array}{l}\text { Endo-1,4-beta- } \\
\quad \text { xylanase }\end{array}$ & $\begin{array}{l}\text { Bacillus } \\
\text { stearothermophilus }\end{array}$ & $\begin{array}{l}\text { MRNVVRKPLTIGLALTLLLP } \\
\text { MGMTATSA }\end{array}$ \\
\hline 21 & P06279 & $\underset{\text { ST }}{\text { AMYBAC }}$ & Alpha-amylase & $\begin{array}{l}\text { Bacillus } \\
\text { stearothermopbilus }\end{array}$ & $\begin{array}{l}\text { MLTFHRIIRKGWMFLLAFL } \\
\text { LTALLFCPTGQPAKA }\end{array}$ \\
\hline 22 & P02932 & $\begin{array}{c}\text { PHOE_EC } \\
\text { OLI }\end{array}$ & $\begin{array}{l}\text { Outer membrane } \\
\text { pore protein } \mathrm{E}\end{array}$ & $\begin{array}{l}\text { Escherichia coli } \\
\text { (strain K12) }\end{array}$ & $\begin{array}{l}\text { MKKSTLALVVMGIVASASVQ } \\
\underline{A}\end{array}$ \\
\hline 23 & P00634 & $\begin{array}{c}\text { PPB_ECO } \\
\text { LI }\end{array}$ & $\begin{array}{c}\text { Alkaline } \\
\text { phosphatase }\end{array}$ & $\begin{array}{l}\text { Escherichia coli } \\
\text { (strain K12) }\end{array}$ & $\begin{array}{l}\text { MKQSTIALALLPLLFTPVTK } \\
\underline{A}\end{array}$ \\
\hline
\end{tabular}




\begin{tabular}{|c|c|c|c|c|c|}
\hline 24 & P0A910 & $\begin{array}{l}\text { OMPA_EC } \\
\text { OLI }\end{array}$ & $\begin{array}{l}\text { Outer membrane } \\
\text { protein A }\end{array}$ & $\begin{array}{l}\text { Escherichia coli } \\
\text { (strain K12) }\end{array}$ & $\begin{array}{l}\text { MKKTAIAIAVALAGEATVA } \\
\text { QA }\end{array}$ \\
\hline 25 & P02931 & $\begin{array}{l}\text { OMPF_EC } \\
\text { OLI }\end{array}$ & $\begin{array}{l}\text { Outer membrane } \\
\text { protein } \mathrm{F}\end{array}$ & $\begin{array}{l}\text { Escherichia coli } \\
\text { (strain K12) }\end{array}$ & $\begin{array}{l}\text { MMKRNILAVIVPALLVAGT } \\
\underline{\text { ANA }}\end{array}$ \\
\hline 26 & P09169 & $\begin{array}{l}\text { OMPT_EC } \\
\text { OLI }\end{array}$ & Protease 7 & $\begin{array}{l}\text { Escherichia coli } \\
\text { (strain K12) }\end{array}$ & MRAKLLGIVLTTPIAISSFA \\
\hline 27 & P06996 & $\begin{array}{l}\text { OMPC_EC } \\
\text { OLI }\end{array}$ & $\begin{array}{l}\text { Outer membrane } \\
\text { protein } C\end{array}$ & $\begin{array}{l}\text { Escherichia coli } \\
\text { (strain K12) }\end{array}$ & $\begin{array}{l}\text { MKVKVLSLLVPALLVAGAA } \\
\text { NA }\end{array}$ \\
\hline 28 & P02943 & $\begin{array}{l}\text { LAMB_EC } \\
\text { OLI }\end{array}$ & Maltoporin & $\begin{array}{l}\text { Escherichia coli } \\
\text { (strain K12) }\end{array}$ & $\begin{array}{l}\text { MMITLRKLPLAVAVAAGVM } \\
\text { SAQAMA }\end{array}$ \\
\hline 29 & POAEX9 & $\begin{array}{c}\text { MALE_EC } \\
\text { OLI }\end{array}$ & $\begin{array}{l}\text { Maltose-binding } \\
\text { periplasmic } \\
\text { protein }\end{array}$ & $\begin{array}{l}\text { Escherichia coli } \\
\text { (strain K12) }\end{array}$ & $\begin{array}{l}\text { MKIKTGARILALSALTTMMF } \\
\text { SASALA }\end{array}$ \\
\hline 30 & P0AEG4 & $\begin{array}{l}\text { DSBA_EC } \\
\text { OLI }\end{array}$ & $\begin{array}{l}\text { Thiol:disulfide } \\
\text { interchange } \\
\text { protein dsbA }\end{array}$ & $\begin{array}{c}\text { Escherichia coli } \\
\text { (strain K12) }\end{array}$ & MKKIWLALAGLVLAFSASA \\
\hline
\end{tabular}

Table 2. In silico analysis of the signal peptides sequences by SignalP 4.1 server.

\begin{tabular}{|c|c|c|c|c|c|c|c|c|c|}
\hline Protein Name & $\begin{array}{c}\mathrm{n}- \\
\text { region }\end{array}$ & $\begin{array}{c}\mathrm{h}- \\
\text { region }\end{array}$ & c-region & $\begin{array}{l}\text { Cleavage } \\
\text { Site }\end{array}$ & $\begin{array}{l}\text { C- } \\
\text { score }\end{array}$ & $\begin{array}{c}\text { Y- } \\
\text { score }\end{array}$ & $\begin{array}{c}\text { S- } \\
\text { score }\end{array}$ & $\begin{array}{c}\mathrm{S}- \\
\text { mean }\end{array}$ & $\begin{array}{c}\text { D- } \\
\text { score }\end{array}$ \\
\hline AMY_BACLI & $\begin{array}{c}1- \\
10(10)\end{array}$ & $\begin{array}{c}11- \\
22(12)\end{array}$ & 24-30(7) & AAA & 0.702 & 0.818 & 0.996 & 0.945 & 0.888 \\
\hline BLAC_BACLI & $\begin{array}{c}1- \\
12(12)\end{array}$ & $\begin{array}{c}13- \\
22(10)\end{array}$ & $23-26(4)$ & ALA & 0.139 & 0.359 & 0.968 & 0.926 & 0.671 \\
\hline SUBF_BACSU & $1-9(9)$ & $\begin{array}{c}10- \\
14(15)\end{array}$ & $15-20(6)$ & AGA & 0.769 & 0.849 & 0.973 & 0.942 & 0.900 \\
\hline CWBA_BACSU & $1-5(5)$ & $\begin{array}{c}6- \\
20(15)\end{array}$ & $20-24(5)$ & SFA & 0.825 & 0.890 & 0.985 & 0.941 & 0.918 \\
\hline YXAL_BACSU & $1-8(8)$ & $\begin{array}{c}9- \\
34(26)\end{array}$ & $35-41(7)$ & AYA & 0.690 & 0.528 & 0.964 & 0.723 & 0.635 \\
\hline YLQB_BACSU & $1-3(3)$ & $\begin{array}{c}4- \\
18(15)\end{array}$ & $19-27(9)$ & $\mathrm{ADA}$ & 0.668 & 0.763 & 0.985 & 0.889 & 0.832 \\
\hline AMY_BACSU & $1-5(5)$ & $\begin{array}{c}6- \\
22(17)\end{array}$ & $23-27(5)$ & AGA & 0.208 & 0.439 & 0.970 & 0.924 & 0.706 \\
\hline PEL_BACSU & $1-3(3)$ & $\begin{array}{c}4- \\
14(11)\end{array}$ & $15-21(7)$ & ANA & 0.613 & 0.735 & 0.961 & 0.880 & 0.814 \\
\hline LYTF_BACSU & $1-4(4)$ & $\begin{array}{c}5- \\
21(17)\end{array}$ & $22-26(5)$ & AEA & 0.724 & 0.774 & 0.905 & 0.828 & 0.803 \\
\hline PPBD_BACSU & $*$ & $*$ & * & * & 0.397 & 0.441 & 0.706 & 0.273 & 0.348 \\
\hline QOX2_BACSU & 1(1) & $\begin{array}{c}2- \\
23(22)\end{array}$ & $24-26(3)$ & LGG & 0.151 & 0.380 & 0.984 & 0.961 & 0.700 \\
\hline GUB_BACSU & $1-3(3)$ & $\begin{array}{c}4- \\
19(16)\end{array}$ & $20-25(6)$ & ASA & 0.676 & 0.798 & 0.993 & 0.939 & 0.876 \\
\hline RNBR_BACAM & $\begin{array}{c}1- \\
11(11)\end{array}$ & $\begin{array}{c}12- \\
32(21)\end{array}$ & $33-34(2)$ & STA & 0.400 & 0.614 & 0.985 & 0.899 & 0.771 \\
\hline SACB_BACAM & $1-5(5)$ & $\begin{array}{c}6- \\
24(19)\end{array}$ & $25-29(5)$ & AFA & 0.761 & 0.799 & 0.936 & 0.848 & 0.826 \\
\hline AMY_BACAM & $1-6(6)$ & $\begin{array}{c}7- \\
23(17)\end{array}$ & $24-28(5)$ & TSA & 0.491 & 0.680 & 0.979 & 0.954 & 0.831 \\
\hline GUB_BACAM & $1-3(3)$ & $\begin{array}{c}4- \\
19(16)\end{array}$ & $20-25(6)$ & VSA & 0.512 & 0.691 & 0.978 & 0.924 & 0.819 \\
\hline DACA_BACST & $1-5(5)$ & $\begin{array}{c}6- \\
23(18)\end{array}$ & $24-30(7)$ & VKA & 0.699 & 0.744 & 0.906 & 0.814 & 0.783 \\
\hline CDGT_BACST & $1-3(3)$ & $\begin{array}{c}4- \\
22(19)\end{array}$ & $23-31(9)$ & VEA & 0.686 & 0.664 & 0.930 & 0.759 & 0.716 \\
\hline
\end{tabular}




\begin{tabular}{|c|c|c|c|c|c|c|c|c|c|}
\hline THER_BACST & $1-4(4)$ & $\begin{array}{c}5- \\
21(17)\end{array}$ & $22-25(4)$ & ASA & 0.792 & 0.852 & 0.969 & 0.890 & 0.873 \\
\hline XYN1_BACST & $1-3(3)$ & $\begin{array}{c}4- \\
23(20)\end{array}$ & $24-28(5)$ & TSA & 0.683 & 0.795 & 0.986 & 0.927 & 0.867 \\
\hline AMY_BACST & $\begin{array}{c}1- \\
10(10)\end{array}$ & $\begin{array}{c}11- \\
26(16)\end{array}$ & $27-33(7)$ & AKA & 0.848 & 0.871 & 0.972 & 0.908 & 0.891 \\
\hline PHOE_ECOLI & $1-4(4)$ & $\begin{array}{c}5- \\
15(11)\end{array}$ & $16-21(6)$ & VQA & 0.169 & 0.390 & 0.960 & 0.881 & 0.660 \\
\hline PPB_ECOLI & $1-4(4)$ & $\begin{array}{c}5- \\
16(12)\end{array}$ & $17-21(5)$ & TKA & 0.426 & 0.618 & 0.944 & 0.874 & 0.759 \\
\hline OMPA_ECOLI & $1-4(4)$ & $5-13(9)$ & $14-21(8)$ & AQA & 0.760 & 0.816 & 0.944 & 0.859 & 0.840 \\
\hline OMPF_ECOLI & $1-4(4)$ & $\begin{array}{c}5- \\
17(13)\end{array}$ & $18-22(5)$ & ANA & 0.786 & 0.847 & 0.959 & 0.918 & 0.886 \\
\hline OMPT_ECOLI & $1-4(4)$ & $5-12(8)$ & $13-20(8)$ & SFA & 0.197 & 0.415 & 0.913 & 0.835 & 0.646 \\
\hline OMPC_ECOLI & $1-4(4)$ & $\begin{array}{c}5- \\
16(12)\end{array}$ & $17-21(5)$ & ANA & 0.542 & 0.708 & 0.982 & 0.929 & 0.829 \\
\hline LAMB_ECOLI & $1-7(7)$ & $8-16(9)$ & $17-25(9)$ & AMA & 0.269 & 0.491 & 0.956 & 0.911 & 0.722 \\
\hline MALE_ECOLI & $1-5(5)$ & $\begin{array}{c}6- \\
20(15)\end{array}$ & $21-26(6)$ & ALA & 0.300 & 0.529 & 0.979 & 0.924 & 0.747 \\
\hline DSBA_ECOLI & $1-3(3)$ & $\begin{array}{l}4-15 \\
(12)\end{array}$ & 16-19(4) & ASA & 0.193 & 0.417 & 0.941 & 0.889 & 0.677 \\
\hline
\end{tabular}

$\mathrm{N}$ region: positively charged $\mathrm{N}$ terminal of SP. H region: aliphatic region of SP. C region: C-terminal region of SP. The S and $\mathrm{C}$-score recognize signal peptide positions and cleavage sites respectively. The combination of C-score and S-score is Y-score, and the average of the S-score is S-mean. D-score (discrimination score) is a simple average of the S-mean and Y-max score.

*: no signal peptide detected.

Table 3. Physicochemical properties of the signal peptides was determined by ProtParam server.

\begin{tabular}{|c|c|c|c|c|c|c|c|c|c|}
\hline $\begin{array}{l}\text { Signal } \\
\text { Peptide }\end{array}$ & $\begin{array}{l}\text { Amino } \\
\text { Acid } \\
\text { Length } \\
\text { (bp) }\end{array}$ & $\begin{array}{l}\text { MW } \\
\text { (Da) }\end{array}$ & $\mathrm{pI}$ & $\begin{array}{c}\text { Net } \\
\text { Positive } \\
\text { Charge }\end{array}$ & $\begin{array}{l}\text { Alipha } \\
\text { tic } \\
\text { Index }\end{array}$ & GRAVY & $\begin{array}{c}\text { Instability } \\
\text { Index of } \\
\text { SP }\end{array}$ & $\begin{array}{c}\text { Instability } \\
\text { Index of } \\
\text { SP+ amyS }\end{array}$ & Solubility \\
\hline $\begin{array}{c}\text { AMY_BA } \\
\text { CLI }\end{array}$ & 29 & $\begin{array}{c}3299 . \\
07\end{array}$ & 11.10 & 4 & 141.72 & 0.752 & $\begin{array}{c}\text { Unstable } \\
(50.51)\end{array}$ & $\begin{array}{l}\text { Stable } \\
(29.94)\end{array}$ & soluble \\
\hline $\begin{array}{c}\text { BLAC_B } \\
\text { ACLI }\end{array}$ & 26 & $\begin{array}{c}2810 . \\
54\end{array}$ & 10.04 & 4 & 131.54 & 1.192 & $\begin{array}{l}\text { Stable } \\
(15.30)\end{array}$ & $\begin{array}{l}\text { Stable } \\
(27.81)\end{array}$ & soluble \\
\hline $\begin{array}{c}\text { SUBF_B } \\
\text { ACSU }\end{array}$ & 30 & $\begin{array}{c}3145 . \\
80\end{array}$ & 12.02 & 5 & 117.00 & 0.497 & $\begin{array}{l}\text { Stable } \\
(20.75)\end{array}$ & $\begin{array}{l}\text { Stable } \\
(28.20)\end{array}$ & soluble \\
\hline$\underset{\text { ACSU }}{\text { CWBA_B }}$ & 25 & $\begin{array}{c}2649 . \\
35\end{array}$ & 8.89 & 2 & 160.00 & 1.596 & $\begin{array}{c}\text { Unstable } \\
(42.97)\end{array}$ & $\begin{array}{l}\text { Stable } \\
(29.37)\end{array}$ & soluble \\
\hline $\begin{array}{c}\text { YXAL_B } \\
\text { ACSU }\end{array}$ & 41 & $\begin{array}{c}3974 . \\
74\end{array}$ & 10.00 & 3 & 105.12 & 0.915 & $\begin{array}{l}\text { Stable } \\
(30.66)\end{array}$ & $\begin{array}{l}\text { Stable } \\
(28.82)\end{array}$ & soluble \\
\hline $\begin{array}{c}\text { YLQB_B } \\
\text { ACSU }\end{array}$ & 27 & $\begin{array}{c}2912 . \\
60\end{array}$ & 7.95 & 1 & 119.63 & 1.122 & $\begin{array}{l}\text { Stable } \\
(31.87\end{array}$ & $\begin{array}{l}\text { Stable } \\
(28.83)\end{array}$ & soluble \\
\hline $\begin{array}{c}\text { AMY_BA } \\
\text { CSU }\end{array}$ & 27 & $\begin{array}{c}3051 . \\
82\end{array}$ & 11.17 & 3 & 137.41 & 1.352 & $\begin{array}{l}\text { Stable } \\
(23.27)\end{array}$ & $\begin{array}{l}\text { Stable } \\
(28.21)\end{array}$ & soluble \\
\hline $\begin{array}{c}\text { PEL_BA } \\
\text { CSU }\end{array}$ & 21 & $\begin{array}{c}2118 . \\
62\end{array}$ & 10.00 & 2 & 111.90 & 0.948 & $\begin{array}{l}\text { Stable } \\
(10.61)\end{array}$ & $\begin{array}{l}\text { Stable } \\
(27.91)\end{array}$ & soluble \\
\hline $\begin{array}{c}\text { LYTF_B } \\
\text { ACSU }\end{array}$ & 26 & $\begin{array}{c}2542 . \\
07\end{array}$ & 9.70 & 2 & 116.54 & 0.769 & $\begin{array}{l}\text { Stable } \\
(0.70)\end{array}$ & $\begin{array}{l}\text { Stable } \\
(27.23)\end{array}$ & soluble \\
\hline $\begin{array}{c}\text { QOX2_B } \\
\text { ACSU }\end{array}$ & 26 & $\begin{array}{c}2843 . \\
68\end{array}$ & 11.00 & 2 & 198.46 & 2.242 & $\begin{array}{l}\text { Stable } \\
(8.20)\end{array}$ & $\begin{array}{l}\text { Stable } \\
(27.45)\end{array}$ & soluble \\
\hline$\underset{\mathrm{CSU}}{\text { GUB_BA }}$ & 28 & $\begin{array}{c}3027 . \\
76\end{array}$ & 9.99 & 2 & 142.86 & 1.443 & $\begin{array}{l}\text { Unstable } \\
(40.54)\end{array}$ & $\begin{array}{l}\text { Stable } \\
(29.31)\end{array}$ & soluble \\
\hline $\begin{array}{c}\text { RNBR_B } \\
\text { ACAM }\end{array}$ & 34 & $\begin{array}{c}3699 . \\
65\end{array}$ & 9.79 & 3 & 129.12 & 1.153 & $\begin{array}{l}\text { Stable } \\
(28.71)\end{array}$ & $\begin{array}{l}\text { Stable } \\
(28.66)\end{array}$ & soluble \\
\hline
\end{tabular}




\begin{tabular}{|c|c|c|c|c|c|c|c|c|c|}
\hline $\begin{array}{c}\text { SACB_B } \\
\text { ACAM }\end{array}$ & 29 & $\begin{array}{c}3008 . \\
61\end{array}$ & 10.30 & 3 & 107.93 & 0.710 & $\begin{array}{l}\text { Stable } \\
(26.18)\end{array}$ & $\begin{array}{l}\text { Stable } \\
(28.52)\end{array}$ & soluble \\
\hline $\begin{array}{c}\text { AMY_BA } \\
\text { CAM }\end{array}$ & 31 & $\begin{array}{c}3582 . \\
47\end{array}$ & 11.74 & 6 & 119.35 & 0.606 & $\begin{array}{c}\text { Unstable } \\
(47.44)\end{array}$ & $\begin{array}{l}\text { Stable } \\
(29.79)\end{array}$ & soluble \\
\hline $\begin{array}{c}\text { GUB_BA } \\
\text { CAM }\end{array}$ & 25 & $\begin{array}{c}2640 . \\
30\end{array}$ & 9.50 & 2 & 148.00 & 1.508 & $\begin{array}{c}\text { Unstable } \\
(45.44)\end{array}$ & $\begin{array}{l}\text { Stable } \\
(29.49)\end{array}$ & soluble \\
\hline $\begin{array}{c}\text { DACA_B } \\
\text { ACST }\end{array}$ & 30 & $\begin{array}{c}3685 . \\
47\end{array}$ & 10.93 & 5 & 91.00 & 0.117 & $\begin{array}{c}\text { Unstable } \\
(86.84)\end{array}$ & $\begin{array}{l}\text { Stable } \\
(32.06)\end{array}$ & soluble \\
\hline $\begin{array}{c}\text { CDGT_B } \\
\text { ACST }\end{array}$ & 31 & $\begin{array}{c}3562 . \\
25\end{array}$ & 8.50 & 1 & 116.13 & 0.887 & $\begin{array}{c}\text { Unstable } \\
(46.86)\end{array}$ & $\begin{array}{l}\text { Stable } \\
(29.76)\end{array}$ & soluble \\
\hline $\begin{array}{c}\text { THER_B } \\
\text { ACST }\end{array}$ & 25 & $\begin{array}{c}2414 . \\
95\end{array}$ & 11.00 & 2 & 121.60 & 1.100 & $\begin{array}{l}\text { Stable } \\
(33.02)\end{array}$ & $\begin{array}{l}\text { Stable } \\
(28.88)\end{array}$ & soluble \\
\hline $\begin{array}{c}\text { XYN1_B } \\
\text { ACST }\end{array}$ & 28 & $\begin{array}{c}2969 . \\
70\end{array}$ & 12.01 & 3 & 128.93 & 0.814 & $\begin{array}{l}\text { Stable } \\
(11.82)\end{array}$ & $\begin{array}{l}\text { Stable } \\
(27.74)\end{array}$ & soluble \\
\hline$\underset{\text { CST }}{\text { AMY_BA }}$ & 34 & $\begin{array}{c}3906 . \\
86\end{array}$ & 11.01 & 4 & 115.00 & 0.876 & $\begin{array}{l}\text { Stable } \\
(35.93)\end{array}$ & $\begin{array}{l}\text { Stable } \\
(29.14)\end{array}$ & soluble \\
\hline $\begin{array}{c}\text { PHOE_E } \\
\text { COLI }\end{array}$ & 21 & $\begin{array}{c}2104 . \\
59\end{array}$ & 10.00 & 2 & 130.00 & 1.195 & $\begin{array}{l}\text { Stable } \\
(1.44)\end{array}$ & $\begin{array}{l}\text { Stable } \\
(27.53)\end{array}$ & soluble \\
\hline $\begin{array}{c}\text { PPB_EC } \\
\text { OLI }\end{array}$ & 21 & $\begin{array}{c}2256 . \\
82\end{array}$ & 10.00 & 2 & 139.52 & 0.971 & $\begin{array}{c}\text { Unstable } \\
(56.02)\end{array}$ & $\begin{array}{l}\text { Stable } \\
(29.80)\end{array}$ & soluble \\
\hline $\begin{array}{c}\text { OMPA_E } \\
\text { COLI }\end{array}$ & 21 & $\begin{array}{c}2046 . \\
50\end{array}$ & 10.00 & 2 & 121.43 & 1.295 & $\begin{array}{l}\text { Stable } \\
(9.52)\end{array}$ & $\begin{array}{l}\text { Stable } \\
(27.86)\end{array}$ & soluble \\
\hline $\begin{array}{c}\text { OMPF_E } \\
\text { COLI }\end{array}$ & 22 & $\begin{array}{c}2266 . \\
83\end{array}$ & 11.00 & 2 & 150.91 & 1.259 & $\begin{array}{c}\text { Unstable } \\
\text { (67.18) }\end{array}$ & $\begin{array}{l}\text { Stable } \\
(30.34)\end{array}$ & soluble \\
\hline $\begin{array}{c}\text { OMPT_E } \\
\text { COLI }\end{array}$ & 20 & $\begin{array}{c}2102 . \\
61\end{array}$ & 11.00 & 2 & 146.50 & 1.290 & $\begin{array}{l}\text { Stable } \\
(2.62)\end{array}$ & $\begin{array}{l}\text { Stable } \\
(27.63)\end{array}$ & soluble \\
\hline $\begin{array}{c}\text { OMPC_E } \\
\text { COLI }\end{array}$ & 21 & $\begin{array}{c}2078 . \\
63\end{array}$ & 10.00 & 2 & 171.90 & 1.552 & $\begin{array}{l}\text { Stable } \\
(14.37)\end{array}$ & $\begin{array}{l}\text { Stable } \\
(28.07)\end{array}$ & soluble \\
\hline $\begin{array}{c}\text { LAMB_E } \\
\text { COLI }\end{array}$ & 25 & $\begin{array}{c}2545 . \\
22\end{array}$ & 11.00 & 2 & 125.20 & 1.332 & $\begin{array}{c}\text { Unstable } \\
(42.97)\end{array}$ & $\begin{array}{l}\text { Stable } \\
(29.37)\end{array}$ & soluble \\
\hline $\begin{array}{c}\text { MALE_E } \\
\text { COLI }\end{array}$ & 26 & $\begin{array}{c}2698 . \\
34\end{array}$ & 11.17 & 3 & 113.08 & 1.012 & $\begin{array}{l}\text { Stable } \\
(2.85)\end{array}$ & $\begin{array}{l}\text { Stable } \\
(27.34)\end{array}$ & soluble \\
\hline $\begin{array}{c}\text { DSBA_E } \\
\text { COLI }\end{array}$ & 19 & $\begin{array}{c}1990 . \\
48\end{array}$ & 10.00 & 2 & 144.21 & 1.416 & $\begin{array}{l}\text { Stable } \\
(11.50)\end{array}$ & $\begin{array}{l}\text { Stable } \\
(28.01)\end{array}$ & soluble \\
\hline
\end{tabular}

MW: molecular weight. pI: isoelectric point. GRAVY: grand average of hydrophobicity.

\section{Secretion pathway and sub-cellular} localization. Predicted secretion pathway for signal peptides connected $\alpha$-amylase are shown in Table 4. All predicted pathways were Sec pathway except for QOX2_BACSU. This signal peptide was ignored for last step. In the last step, all remained signal peptides fusions were evaluated as secreted proteins.

\section{DISCUSSION}

Recombinant protein secretion to the extracellular space includes several important privileges in comparison to intracellular expression approaches, including high stability and solubility of the product, enhanced biological activity and simplified downstream processing. Secretion of a specific protein into the extracellular space can be mediated via an expression system by addition a signal peptide to the $\mathrm{N}$-terminal of a protein (Anné et al., 2016, Song et al., 2015). The signal peptide is considered as a momentous factor that has an essential effect on the secretion pathway selection and consequently, on the yield of the recombinant protein. So, secretion of the recombinant protein can be enhanced by the choice of an efficient signal peptide (Guan et al., 2016). 
Table 4. Secretion sorting and sub-cellular location of signal peptides

\begin{tabular}{|c|c|c|c|c|c|c|}
\hline \multirow[b]{2}{*}{ Signal peptides } & \multicolumn{2}{|c|}{ Secretion Pathway } & \multicolumn{4}{|c|}{ Sub-cellular localization } \\
\hline & $\begin{array}{c}\text { Type of } \\
\text { SP }\end{array}$ & $\begin{array}{l}\text { Reliability } \\
\text { Score (\%) }\end{array}$ & Cytoplasmic & Membrane & $\begin{array}{c}\text { Secreted } \\
\text { (extracellular) }\end{array}$ & $\begin{array}{c}\text { Final } \\
\text { prediction } \\
\text { site }\end{array}$ \\
\hline AMY_BACLI & $\begin{array}{c}\text { Sec } \\
\text { pathway }\end{array}$ & 0.995 & 3.36 & 0.00 & 6.63 & Secreted \\
\hline BLAC_BACLI & $\begin{array}{c}\text { Sec } \\
\text { pathway }\end{array}$ & 0.994 & 3.35 & 0.05 & 6.58 & Secreted \\
\hline SUBF_BACSU & $\begin{array}{c}\text { Sec } \\
\text { pathway }\end{array}$ & 1.000 & 3.32 & 0.05 & 6.61 & Secreted \\
\hline CWBA_BACSU & $\begin{array}{c}\text { Sec } \\
\text { pathway }\end{array}$ & 1.000 & 3.34 & 0.01 & 6.63 & Secreted \\
\hline YXAL_BACSU & $\begin{array}{c}\text { Sec } \\
\text { pathway }\end{array}$ & 0.937 & 3.32 & 0.15 & 6.50 & Secreted \\
\hline YLQB_BACSU & $\begin{array}{c}\text { Sec } \\
\text { pathway }\end{array}$ & 0.993 & 3.33 & 0.04 & 6.62 & Secreted \\
\hline AMY_BACSU & $\begin{array}{c}\text { Sec } \\
\text { pathway }\end{array}$ & 0.990 & 3.36 & 0.03 & 6.59 & Secreted \\
\hline PEL_BACSU & $\begin{array}{c}\text { Sec } \\
\text { pathway }\end{array}$ & 1.000 & 3.32 & 0.01 & 6.65 & Secreted \\
\hline LYTF_BACSU & $\begin{array}{c}\text { Sec } \\
\text { pathway }\end{array}$ & 1.000 & 3.33 & 0.04 & 6.62 & Secreted \\
\hline QOX2_BACSU & $\begin{array}{c}\text { TM } \\
\text { segment }\end{array}$ & 0.810 & * & $*$ & $*$ & $*$ \\
\hline GUB_BACSU & $\begin{array}{c}\text { Sec } \\
\text { pathway }\end{array}$ & 0.999 & 3.35 & 0.00 & 6.63 & Secreted \\
\hline RNBR_BACAM & $\begin{array}{c}\text { Sec } \\
\text { pathway }\end{array}$ & 0.986 & 3.36 & 0.02 & 6.61 & Secreted \\
\hline SACB_BACAM & $\begin{array}{c}\text { Sec } \\
\text { pathway }\end{array}$ & 1.000 & 3.29 & 0.52 & 6.10 & Secreted \\
\hline AMY_BACAM & $\begin{array}{c}\text { Sec } \\
\text { pathway }\end{array}$ & 0.999 & 3.36 & 0.00 & 6.63 & Secreted \\
\hline GUB_BACAM & $\begin{array}{c}\text { Sec } \\
\text { pathway }\end{array}$ & 1.000 & 3.32 & 0.00 & 6.67 & Secreted \\
\hline DACA_BACST & $\begin{array}{c}\text { Sec } \\
\text { pathway }\end{array}$ & 1.000 & 3.32 & 0.00 & 6.66 & Secreted \\
\hline CDGT_BACST & $\begin{array}{c}\text { Sec } \\
\text { pathway }\end{array}$ & 0.993 & 3.31 & 0.04 & 6.62 & Secreted \\
\hline THER_BACST & $\begin{array}{c}\text { Sec } \\
\text { pathway }\end{array}$ & 1.000 & 3.32 & 0.00 & 6.67 & Secreted \\
\hline XYN1_BACST & $\begin{array}{c}\text { Sec } \\
\text { pathway }\end{array}$ & 0.997 & 3.33 & 0.00 & 6.65 & Secreted \\
\hline AMY_BACST & $\begin{array}{c}\text { Sec } \\
\text { pathway }\end{array}$ & 1.000 & 3.32 & 0.00 & 6.66 & Secreted \\
\hline PHOE_ECOLI & $\begin{array}{c}\text { Sec } \\
\text { pathway }\end{array}$ & 1.000 & 3.32 & 0.12 & 6.54 & Secreted \\
\hline PPB_ECOLI & $\begin{array}{c}\text { Sec } \\
\text { pathway }\end{array}$ & 1.000 & 3.32 & 0.15 & 6.50 & Secreted \\
\hline OMPA_ECOLI & $\begin{array}{c}\text { Sec } \\
\text { pathway }\end{array}$ & 1.000 & 3.33 & 0.12 & 6.52 & Secreted \\
\hline OMPF_ECOLI & $\begin{array}{c}\text { Sec } \\
\text { pathway }\end{array}$ & 1.000 & 3.32 & 0.07 & 6.59 & Secreted \\
\hline OMPT_ECOLI & $\begin{array}{c}\text { Sec } \\
\text { pathway }\end{array}$ & 1.000 & 3.32 & 0.10 & 6.55 & Secreted \\
\hline
\end{tabular}




\begin{tabular}{ccccccc}
\hline OMPC_ECOLI & $\begin{array}{c}\text { Sec } \\
\text { pathway } \\
\text { Sec }\end{array}$ & 0.999 & 3.32 & 0.14 & 6.51 & Secreted \\
LAMB_ECOLI & $\begin{array}{c}1.000 \\
\text { pathway } \\
\text { Sec } \\
\text { MALE_ECOLI }\end{array}$ & 1.000 & 3.32 & 0.03 & 6.63 & Secreted \\
DSBAthway \\
$\begin{array}{c}\text { Sec } \\
\text { pathway }\end{array}$ & 1.000 & 3.32 & 0.03 & 6.63 & Secreted \\
\hline
\end{tabular}

Today, several online tools are used for identification of signal peptides to reduce the cost and improving the quality of experimental investigation (Gasteiger et al., 2005, Magnan et al., 2009, Petersen et al., 2011). For this purpose, in this study, 30 prokaryotic signal peptides were selected. These signal peptides have illustrated positive effects on secretion of some proteins in previous investigations (Watanabe et al., 2009, Chen et al., 2015, Degering et al., 2010). Then numerous online servers such as SignalP, ProtParam, and SOLpro are used for prediction of the presence and cleavage site location, physico-chemical properties and protein solubility of signal peptides. These factors are important to improve the protein secretion levels.

In this study, for the prediction of cleavage site presence and its location on the signal peptide sequences SignalP server was applied. The graphical output from SignalP comprises three different scores, C, S and Y, The S and C-score recognize signal peptide positions and cleavage sites respectively. The combination of $\mathrm{C}$-score and $\mathrm{S}$-score is $\mathrm{Y}$-score, and the average of the $\mathrm{S}$ score is $\mathrm{S}$-mean. D-score (discrimination score) is a simple average of the S-mean and Y-max score and when a value $>0.57$ represent a secretory protein and value of $<0.57$ is interpreted as a nonsecretory protein. As shown in Table 2, D-scores of the PPBD_BACSU was under the cutoff (0.57), which indicated that the cleavage site of it cannot appropriately be recognized by peptidase enzyme. Amino acids with small neutral side chains like alanine in the -3 and the -1 position of c-region are needed to correct cleavage of the signal peptide. The important sites of the $\mathrm{C}$-region are position 1 and 3 before the cleavage site that is recognized as the $(-3,-1)$ rule or AXA motif. This motif is universal in prokaryotes, eukaryotes organelles SPs. Table 2 shows that most of the signal peptides had a proper cleavage site. Ala amino acid is conserved at positions -3 and -1 .
Replacing of Ala at these positions with other residues, specifically at position -1 , disturbs the cleavage site (Owji et al., 2018). Exceptionally, as PPBD_BACSU have a D-scores 0.348, it is not applicable for the secretion of the $\alpha$-amylase protein.

To design a secreted protein properly, structural and physicochemical features of the signal peptides should be considered. Numerous bioinformatics servers are provided to anticipate and analyze the physicochemical properties of signal peptides. In the present study, for investigation of the structural and physicochemical features of different signal peptides, ProtParam online server was used. The results were included in molecular weight, amino acid length, net positive charge, instability and aliphatic indexes, pI and GRAVY (Gasteiger et al., 2005).

Improving an applicable signal peptide is depending on the $\mathrm{n}$ region with positively charged amino acids. Positively charged amino acids interacts with the membrane phospholipids and it would be an advantage. Therefore, if one or more basic amino acids present in the signal peptide $n$ region, it may be more useful. Regarding these facts, when the net positive charge of the $n$ region lowered to zero or get a negative value the rate of transportation across the membrane can significantly decrease. (Freudl, 2018, Owji et al., 2018). The net positive charge for most of the studied signal peptides was +2 , and AMY_BACAM had the highest net positive charge in this study.

The h-region's hydrophobicity is essential for transferring of the protein out of the membrane. So elevated hydrophobicity levels and increased h-region length can help to improve the protein secretion. On the other hand, a polar or charged amino acid residue can disturb the core hydrophobicity and reduces or stops 
transportation across the membrane (Chen et al., 1996).

To study the hydrophobicity of the adopted signal peptides, GRAVY and aliphatic index were measured. The increase in GRAVY and aliphatic index means high hydrophobicity. The grand average of hydrophobicity (GRAVY) protein is the sum of hydrophobicity values of all the amino acid residues and dividing by the number of residues in the sequence (Kyte and Doolittle, 1982). The aliphatic index is interpreted as the relative volume taken up by aliphatic side chains (valine, leucine, isoleucine, and alanine) (Ikai, 1980). According to the values of aliphatic index and GRAVY that are shown in Table 3, the hydrophobicity amounts of the signal peptides, QOX2_BACSU, OMPC_ECOLI and CWBA_BACSU were the highest levels among all 30 signal peptides that could interact with hydrophobic interior side of the membrane transporter proteins.

Protein stability that is the sum of all forces predict that a protein in the experimental stages remain in its correct conformation or it becomes misfolded. Generally, the protein stability is affected by hydrophobic interactions, hydrogen bonds, and conformational entropy and the fate of unstable proteins is usually to be degraded using ATP-dependent cytosolic systems in both bacterial and eukaryotic cells (Goldenzweig and Fleishman, 2018, Gur and Sauer, 2008). Based on ProtParam, instability index reflects the instability of the signal peptides linking to $\alpha$-amylase protein. If instability index of a protein is to be lower than 40 (instability index $<40$ ), it will be stable, a value of $>40$ can be an indication of unstable protein (Guruprasad et al., 1990). The results showed $\alpha$ amylase fused to all signal peptides were predicted to be stable.

In the experimental stages of a study, protein insolubility can produce some difficulties, so accurate analysis of the protein solubility is necessary. An insoluble protein has more exposed hydrophobic regions which can interact with each other and cause protein aggregation. A lot of experimental investigations have been done to get the soluble form of recombinant proteins, including chaperone co-expression, culture temperature regulation, efficient host strain and vector that require long time trial-and-error approaches (Rosano and Ceccarelli, 2014). Thus, the in-silico approaches are essential for analyzing protein solubility. Among different available servers, SOLpro is a sequence-based solubility predictor tool that has an accuracy value of $74 \%$ (Magnan et al., 2009). In the present study, SOLpro online server was used for analysis of overexpressed $\alpha$-amylase solubility when linked to the various signal peptides in B.subtilis. According to Table 4, $\alpha$-Amylase was predicted to be soluble in fusion with all the signal peptides.

Protein export pathways in B. subtilis are twin-arginine translocation (Tat) pathway, Sec pathway and ATP-binding cassette (ABC) pathway. However Sec and Tat pathways are used most commonly for recombinant protein secretion in B. subtilis. In the Sec pathway unfolded proteins and in the Tat pathway folded proteins across the cytoplasmic membrane. So, it seems secretion recombinant proteins folding in Tat pathway causes protein aggregation and making inclusion bodies. So, secretion of proteins in unfolded state in the sec pathway is more appropriate (van Dijl and Hecker, 2013, Rusch and Kendall, 2007, Natale et al., 2008). Except QOX2_BACSU that may translocate into transmembrane segment, all signal peptides were predicted to direct $\alpha$-amylase through $\mathrm{Sec}$ pathway. Ultimately, all remained signal peptides may be useful for the secretory production and can target protein into the culture medium with a reliability score more than 0.6 (Table 4 ).

\section{CONCLUSION}

Finally, GUB_BACAM and THER_BACST can be useful for the future practical investigations in $\alpha$-amylase secretion. In silico investigations and analysis of signal peptides using online tools are acceptable, but these results need to be approved by experimental analysis.

\section{ACKNOWLEDGEMENTS}

This study was financially supported by the office of vice-chancellor for research of Shiraz University of Medical Sciences (Grant No. 98-0174-19799). 


\section{REFERENCES}

Anné, J., Economou, A., \& Bernaerts, K. 2016. Protein secretion in Gram-positive bacteria: from multiple pathways to biotechnology. Protein and Sugar Export and Assembly in Gram-positive Bacteria. pp. 267-308. Springer, Cham.

Azzopardi, E., Lloyd, C., Teixeira, S. R., Conlan, R. S., \& Whitaker, I. S. 2016. Clinical applications of amylase: Novel perspectives. Surgery 160(1): 26-37.

Bagos, P. G., Nikolaou, E. P., Liakopoulos, T. D., \& Tsirigos, K. D. 2010. Combined prediction of Tat and Sec signal peptides with hidden Markov models. Bioinformatics 26(22): 2811-7.

Chen, H., Kim, J., \& Kendall, D. A. 1996. Competition between functional signal peptides demonstrates variation in affinity for the secretion pathway. Journal of Bacteriology 78(23): 665864.

Chen, J., Gai, Y., Fu, G., Zhou, W., Zhang, D., \& Wen, J. 2015. Enhanced extracellular production of $\alpha$-amylase in Bacillus subtilis by optimization of regulatory elements and overexpression of PrsA lipoprotein. Biotechnology Letters 37(4): 899-906.

Cui, Y., Meng, Y., Zhang, J., Cheng, B., Yin, H., Gao, C., Xu, P., \& Yang, C. 2017. Efficient secretory expression of recombinant proteins in Escherichia coli with a novel actinomycete signal peptide. Protein Expression and Purification 129: 69-74.

Degering, C., Eggert, T., Puls, M., Bongaerts, J., Evers, S., Maurer, K. H., \& Jaeger, K. E. 2010. Optimization of protease secretion in Bacillus subtilis and Bacillus licheniformis by screening of homologous and heterologous signal peptides. Applied and Environmental Microbiology 76(19): 6370-6.

Freudl, R. 2018. Signal peptides for recombinant protein secretion in bacterial expression systems. Microbial Cell Factories 17(1): 52.

Gasteiger, E., Hoogland, C., Gattiker, A., Wilkins, M. R., Appel, R. D., \& Bairoch, A. 2005. Protein identification and analysis tools on the ExPASy server. The proteomics protocols handbook. pp. 571-607. Springer.

Goldenzweig, A. \& Fleishman, S. J. 2018. Principles of protein stability and their application in computational design. Annual Review of Biochemistry 87: 105-29.

Green, E. R. \& Mecsas, J. 2016. Bacterial Secretion Systems: An Overview. Microbiology Spectrum 4(1).

Guan, C., Cui, W., Cheng, J., Liu, R., Liu, Z., Zhou, L., \& Zhou, Z. 2016. Construction of a highly active secretory expression system via an engineered dual promoter and a highly efficient signal peptide in Bacillus subtilis. New Biotechnology 33(3): 372-9.

Gur, E. \& Sauer, R. T.2008. Recognition of misfolded proteins by Lon, a AAA+ protease. Genes \& Development 22(16): $2267-$ 77.

Guruprasad, K., Reddy, B. B., \& Pandit, M. W. 1990. Correlation between stability of a protein and its dipeptide composition: a novel approach for predicting in vivo stability of a protein from its primary sequence. Protein Engineering, Design and Selection 4(2): 155-61.

Ikai, A. 1980. Thermostability and aliphatic index of globular proteins. The Journal of Biochemistry 88(6): 1895-8.

Kindle, K. L. 1983. Characteristics and production of thermostable $\alpha$-amylase. Applied Biochemistry and Biotechnology 8(2): 153-70.

Kyte, J. \& Doolittle, R. F. 1982. A simple method for displaying the hydropathic character of a protein. Journal of Molecular Biology 157(1): 105-32.
Li, L. \& Somerset, S. 2014. Digestive system dysfunction in cystic fibrosis: challenges for nutrition therapy. Digestive and Liver Disease 46(10): 865-74.

Magnan, C. N., Randall, A., \& Baldi, P. 2009. SOLpro: accurate sequence-based prediction of protein solubility. Bioinformatics 25(17): 2200-7.

Mehta, D. \& Satyanarayana, T. 2016. Bacterial and Archaeal $\alpha$ Amylases: Diversity and Amelioration of the Desirable Characteristics for Industrial Applications. Frontiers in Microbiology 7: 1129.

Mobini-Dehkordi, M. \& Javan, F. A. 2012. Application of alphaamylase in biotechnology. Journal of Biology and Today's world 1: 39-50.

Mohammadi, S., Mostafavi-Pour, Z., Ghasemi, Y., Barazesh, M., \& Pour, S. K. 2018. In silico Analysis of Different Signal Peptides for the Excretory Production of Recombinant NS3-GP96 Fusion Protein in Escherichia coli. International Journal of Peptide Research and Therapeutics: 1-12.

Mousavi, P., Mostafavi-Pour, Z., Morowvat, M. H., Nezafat, N. Zamani, M., Berenjian, A., \& Ghasemi, Y. 2017. In silico analysis of several signal peptides for the excretory production of reteplase in Escherichia coli. Current Proteomic. 14(4): 326-35

Nascimento, B. M. 2013. Production of recombinant a-amylase from Bacillus subtilis by Kluyveromyces lactis.

Natale, P., Brüser, T., \& Driessen, A. J. 2008. Sec-and Tatmediated protein secretion across the bacterial cytoplasmic membrane-distinct translocases and mechanisms. Biochimica et Biophysica Acta (BBA)-Biomembranes 1778(9): 1735-56.

Negahdaripour, M., Nezafat, N., Hajighahramani, N., \& Soheil Rahmatabadi, S. 2017. In silico study of different signal peptides for secretory production of interleukin-11 in Escherichia coli. Current Proteomics 14(2): 112-21.

Owji, H., Nezafat, N., Negahdaripour, M., Hajiebrahimi, A., \& Ghasemi, Y. 2018. A Comprehensive Review of Signal Peptides: Structure, Roles, and Applications. European Journal of Cell Biology 97(6): 422-41.

Petersen, T. N., Brunak, S., Von Heijne, G., \& Nielsen, H. 2011. SignalP 4.0: discriminating signal peptides from transmembrane regions. Nature Methods 8(10): 785.

Rosano, G. L. \& Ceccarelli, E. A. 2014. Recombinant protein expression in Escherichia coli: advances and challenges. Frontiers in Microbiology 5: 172.

Rusch, S. L. \& Kendall, D. A. 2007. Interactions that drive Secdependent bacterial protein transport. Biochemistry 46(34): 9665-73.

Schallmey, M., Singh, A., \& Ward, O. P. 2004. Developments in the use of Bacillus species for industrial production. Canadian journal of Microbiology 50(1): 1-7.

Singh, R., Kumar, M., Mittal, A., \& Mehta, P. K. 2016. Microbial enzymes: industrial progress in 21st century. 3 Biotech 6(2): 174.

Song, Y., Nikoloff, J. M., \& Zhang, D. 2015. Improving protein production on the level of regulation of both expression and secretion pathways in Bacillus subtilis. Journal of Microbiology and Biotecbnology 25(7): 963-77.

Taheri-Anganeh, M., Khatami, S. H., Jamali, Z., Savardashtaki, A., Ghasemi, Y., \& Mostafavipour, Z. 2019. In silico analysis of suitable signal peptides for secretion of a recombinant alcohol dehydrogenase with a key role in atorvastatin enzymatic synthesis. Molecular Biology Research Communications 8(1): 17-26.

Tjalsma, H., Antelmann, H., Jongbloed, J. D., Braun, P. G., Darmon, E., Dorenbos, R., Dubois, J. Y. F., Westers, H., Zanen, G., Quax, W. J., \& Kuipers, O.P. 2004. Proteomics of protein secretion by Bacillus subtilis: separating the 
"secrets" of the secretome. Microbiology and Molecular Biology Reviews 68(2): 207-33.

van Dijl, J. \& Hecker, M. 2013. Bacillus subtilis: from soil bacterium to super-secreting cell factory. Microbial Cell Factories 3.

Wang, P., Qin, W., Xu, J., Yan, Y., Tian, J., Wu, N., \& Yao, B. 2016. Enhancing the soluble expression of an amylase in Escherichia coli by the mutations related to its domain interactions. Protein Expression and Purification 120: 35-41.

Watanabe, K., Tsuchida, Y., Okibe, N., Teramoto, H., Suzuki, N., Inui, M., \& Yukawa, H. 2009. Scanning the Corynebacterium glutamicum R genome for high-efficiency secretion signal sequences. Microbiology 155(3): 741-50.

Zobel, S., Kumpfmüller, J., Süssmuth, R. D., \& Schweder, T. 2015. Bacillus subtilis as heterologous host for the secretory production of the non-ribosomal cyclodepsipeptide enniatin. Applied Microbiology and Biotechnology 99(2): 681-91. 